\title{
Diagnostic Value of Contrast-enhanced Digital Mammography Versus Contrast-enhanced MRI for Detecting Residual Disease after Neoadjuvant Chemotherapy for Breast Cancer
}

\section{Eun Young Kim}

Kangbuk Samsung Hospital, Sungkyunkwan University School of Medicine

Ji-Sup Yun

Kangbuk Samsung Medical Center

Yong Lai Park

Kangbuk Samsung Medical Center

Chan Heun Park

Kangbuk Samsung Medical Center

Mi-Ri Kwon

Kangbuk Samsung Medical Center

Ju Hee Moon

Kangbuk Samsung Medical Center

Inyoung Youn

Kangbuk Samsung Medical Center

Yoon Jung Choi

Kangbuk Samsung Medical Center

Shin Ho Kook ( $\nabla$ gimo.kim@samsung.com )

Kangbuk Samsung Hospital, Sungkyunkwan University School of Medicine

Research article

Keywords: Breast neoplasms, Contrast media, Magnetic resonance imaging, Mammography,

Neoadjuvant

Posted Date: October 27th, 2020

DOI: https://doi.org/10.21203/rs.3.rs-65472/v1

License: (c) (i) This work is licensed under a Creative Commons Attribution 4.0 International License.

Read Full License 


\section{Abstract}

Background: Preoperative evaluation of breast cancer using contrast-enhanced digital mammography (CEDM) as a possible alternative to contrast-enhanced magnetic resonance imaging (CEMRI) has gained acceptance. Our aim is to compare the diagnostic performance of CEDM and CEMRI for chemotherapeutic response in breast cancer patients who underwent neoadjuvant chemotherapy (NAC).

Methods: From November 2017 to October 2018, 30 patients with invasive carcinoma who performed both CEDM and CEMRI were included. Residual malignancy sizes after NAC were compared with histopathological results. The diagnostic performances for detecting residual cancers were compared using Lin concordance and Pearson correlation coefficients.

Results: Thirty patients were included for the analysis. Mean tumor size after NAC was $1.22 \mathrm{~cm}$ (range: $0-7.0 \mathrm{~cm}$ ) for CEDM and $1.13 \mathrm{~cm}$ (range: $0-5.1 \mathrm{~cm}$ ) for CEMRI compared with $1.89 \mathrm{~cm}$ (range: $0-12.0$ $\mathrm{cm})$ at final pathology measurement.

Sensitivity for identifying residual lesion was for CEDM and CEMRI is as follows (CEDM $62.5 \%, 95 \% \mathrm{Cl}$ 40.6-81.2, CEMRI 66.7\%, 95\% Cl 44.7-84.4). The positive predictive value (PPV) for residual lesion was 93.8\% (95\% $\mathrm{Cl} 69.8-99.8)$ for CEDM and 88.9\% (95\% $\mathrm{Cl} 65.3-98.6)$ for CEMRI. CEDM had a mean difference from pathology measurement of $0.668 \mathrm{~cm}$, with a concordance coefficient of 0.202 and a Pearson correlation coefficient of $0.231(p=0.220)$.

Conclusions: Diagnostic value of CEDM for detecting residual tumor extent after NAC was comparable to that of CEMRI.

\section{Background}

Neoadjuvant chemotherapy (NAC) is systemic therapy applying to patients with breast cancer, especially large inoperable cancer. NAC downsizes primary tumor, which increases the chances of breast-conserving therapy. Evaluating tumor response to NAC is important for speculating long-term outcomes and future treatment plans. Contrast-enhanced magnetic resonance imaging (CEMRI), ultrasonography (US), and mammography are imaging modalities used to predict chemotherapeutic response.

Alongside development of digital mammography, Lewin et al established contrast-enhanced digital mammography (CEDM) in 2003 [1]. CEDM traces uptake of iodine-based contrast agent in breast tissue. Past studies have showed that CEDM yields valuable information on shape and vascular enhancement, concordant with CEMRI [2]. CEDM showed better sensitivity of breast cancer detection with high specificity due to higher contrast and better lesion delineation than mammography alone, even in dense breasts [3]. CEMRI shows low specificity for breast cancer detection, high false-positive (FP) rate and leading to overdiagnosis and overtreatment of breast cancer [4]. 
The preoperative role of CEDM as a possible alternative to CEMRI has gained acceptance. Recently published clinical results have reported that CEDM feasibility for detecting primary cancers was similar to that of CEMRI [5]. However, few studies have compared the diagnostic performance and effects of CEDM and CEMRI for evaluating remnant lesion after NAC in breast cancer [6, 7].

Our aim is to compare the diagnostic performance of CEDM and CEMRI for assessing extent of remnant lesion in breast cancer patients who received NAC and to determine the accuracy of CEDM and CEMRI for predicting pathologic complete response (pCR).

\section{Methods}

\section{Patient selection}

From November 2017 to October 2018, 340 patients were diagnosed as primary breast cancer at 00 Hospital, and 53 patients underwent NAC. Patients who were 18 years or older and histologically proven breast cancer with indications for NAC were included. Patients were excluded if pregnant or had anaphylactic reaction to any contrast agent according to the guidelines of the Korean Society of Radiology [8]. This prospective study was approved by the Institutional Review Board (00000 2017-09027). The use of iodinated contrast material was approved by Korean Food and Drug Administration. Informed consent was obtained from all patients. Patient's clinical information was collected.

\section{Contrast-enhanced digital mammography and contrast- enhanced magnetic resonance imaging techniques}

Mammography was carried out before and after NAC using a full-field digital mammography unit (Lorad Selenia; Hologic, Danbury, USA) using standard craniocaudal (CC) and mediolateral oblique (MLO) views. US examinations (iU22 platform, Philips Healthcare, Bothell, USA; Aixplorer, SuperSonic Imagine, Aix-enProvence, France) using a 5-12 and 5-15 MHz linear transducer were carried out before and after NAC.

CEDM and CEMRI were performed before and after NAC. All CEDM examinations were performed within 7 days of CEMRI using a digital mammography device capable of dual energy CEDM acquisition (Selenia Dimensions; Hologic, Bedford, USA). Previously, techniques of CEDM and CEMRI were described in detail $[9,10]$.

\section{Interpretation of contrast-enhanced digital mammography and contrast-enhanced magnetic resonance imaging}

All US, mammography, and CEMRI reports were interpreted using the American College of Radiology (ACR) Breast Imaging Reporting and Data System (BI-RADS) by five breast imaging radiologists with 624 years of experience [11]. All patients were examined first with CEMRI, followed by CEDM. The median interval between CEDM and CEMRI was 5.1 days (range: 1-29 days). CEDM results were reviewed by a radiologist who was blinded to the CEMRI results. The radiologist was blinded to the results of other 
studies when reviewing CEDM and CEMRI. During CEMRI interpretation, when there is discordant result, one radiologist reviewed CEMRI and reached consensus. The largest diameter of the contrast-enhancing lesion was correlated and categorized according to the response evaluation criteria in solid tumors (RECIST) before and after NAC [12]. In cases with multiple tumors, the largest tumor was used to measure size. Previously, method of radiologic response was described in detail [13].

\section{Histopathological analysis}

We obtained carcinoma tissue before NAC using core needle biopsies. The formalin-fixed paraffinembedded specimens were cut into 3- $\mu$ m-thick sections and stained with hematoxylin and eosin for microscopic evaluation. Immunohistochemical staining was performed on $3 \mu \mathrm{m}$-thick TMA block sections. Estrogen receptor (ER) and progesterone receptor (PR) status was assessed using the Allred scoring method [14]. Human epidermal growth factor receptor-2 (HER-2) overexpression was evaluated using American Society of Clinical Oncology/College of American Pathologists guideline recommendations. In cases with equivocal HER-2 staining (score 2), silver in situ hybridization (Ventana Medical Systems, Tucson, AZ, USA) was performed to determine HER2 gene status. Tumor response was defined as follows; pathologic complete response ( $\mathrm{PCR}$ ) was defined as absence of residual invasive carcinoma in resected breast specimens (ypT0/is) [15]. Pathologic tumor response was categorized according to RECIST criteria [12]. In non-pCR cases, the largest histopathologic diameters of residual tumors were measured.

\section{Statistical analysis}

In the absence of an a priori hypothesis, calculation of sample size could not be performed. Categorical data are summarized as frequencies and percentages. Tumor sizes measured by CEDM and CEMRI were compared to those measured on pathology (reference standard) within a range of $-1 \mathrm{~cm}-1 \mathrm{~cm}$.

Agreements in histological size between CEDM and CEMRI measurements were evaluated using BlandAltman plots. Lin's concordance and Pearson correlation coefficient were estimated to assess agreement between CEDM, CEMRI and histopathologic results. Sensitivity (true positive (TP) rate) was defined as the probability by which a no-pCR can be detected by CEDM or CEMRI (TP/(TP + false negative (FN))); specificity (true negative (TN) rate) was defined as probability by which a pCR can be detected by CEDM or CEMRI (TN/(false positive (FP) + TN)); positive predictive value (PPV) was defined as probability by which no-radiologic $\mathrm{CR}(\mathrm{rCR})$ predicts no-pCR $(\mathrm{TN} /(\mathrm{FN}+\mathrm{TN})$; and negative predictive value (NPV) was defined as probability by which $r C R$ predicts $\mathrm{PCR}(\mathrm{TP} /(\mathrm{TP}+\mathrm{FP}))$. The results were calculated with corresponding 95\% confidence intervals (Cls).The McNemar's test was used to determine differences between CEDM and CEMRI results. A p-value $<0.05$ (2-tailed) was considered statistically significant. All statistical analyses were performed with PASW Statistics for Windows, Version 18.0 (SPSS Inc., Chicago, IL, USA).

\section{Results}

\section{Patient and tumor characteristics}


Of the initial 36 patients enrolled, 30 completed the study ( 3 were lost during follow-up, 3 dropped because of an allergic reaction after injection of contrast medium). Thirty women with breast cancer were included in the analysis (Table 1). Mean [ \pm standard deviation (SD)] age at the time of enrollment was $46.2 \pm 8.5$ years (range: $31-60$ years). Five of the 29 (17.2\%) patients had a family history of breast cancer. All 30 breast cancers had invasive ductal carcinoma (IDC), not otherwise specified (NOS) on initial core needle biopsy. Six tumors (20.0\%) were hormone receptor (HR)+/ HER2-, 10 (33.3\%) were HR+/HER2+, 5 (16.7\%) were HR-/HER2+, and 9 (30.0\%) were HR-/HER2-. According to the RECIST criteria, (11) 6 patients achieved pCR, 19 achieved pPR, three showed pSD, and two showed pPD. Pathologic complete response was noted in 6 cases $(20.0 \%)$, all of which were IDC: $4(66.7 \%)$ were triple negative and 2 (33.3\%) were luminal HER2 type. Radiologic CR was observed in 14 cases according to CEDM $(46.7 \%)$ and 12 according to CEMRI (40.0\%). Among these 6 pCR cases, two were positive according to both CEDM and CEMRI. Regarding correlations with histopathological results, CEDM defined 27 patients as responders ( $14 \mathrm{CR}+13 \mathrm{PR})$, and CEMRI defined 26 patients as responders (12 CR $+14 \mathrm{PR})$. 
Table 1

Clinicopathological characteristics of patients

\begin{tabular}{|c|c|}
\hline Characteristics & Value \\
\hline Age (years) & $46.2(8.5)$ \\
\hline Postmenopausal & $11(37.9)$ \\
\hline Family history of breast cancer & $5(17.2)$ \\
\hline \multicolumn{2}{|l|}{ Histologic type before NAC } \\
\hline IDC & $30(100.0)$ \\
\hline \multicolumn{2}{|c|}{ Immunohistochemical subtype before NAC } \\
\hline $\mathrm{HR}^{+} / \mathrm{HER}^{-}$ & $6(20.0)$ \\
\hline $\mathrm{HR}^{+} / \mathrm{HER}^{+}$ & 10 (33.3) \\
\hline $\mathrm{HR}^{-} / \mathrm{HER}^{+}$ & 5 (16.7) \\
\hline $\mathrm{HR}^{-} / \mathrm{HER}^{-}$ & $9(30.0)$ \\
\hline \multicolumn{2}{|l|}{ NAC regimen } \\
\hline AC followed by $T$ & $14(46.7)$ \\
\hline TCHP & $13(43.3)$ \\
\hline AT & $3(10.0)$ \\
\hline \multicolumn{2}{|l|}{ Type of surgery } \\
\hline Total mastectomy & $17(56.7)$ \\
\hline Partial mastectomy & $13(43.3)$ \\
\hline \multicolumn{2}{|l|}{ Histologic type after NAC } \\
\hline IDC & $10(41.7)$ \\
\hline IDC + DCIS & $10(41.7)$ \\
\hline DCIS & $4(16.6)$ \\
\hline \multicolumn{2}{|l|}{ Number of cancer lesions after NAC } \\
\hline None (pCR) & $6(20.0)$ \\
\hline Single & $19(63.3)$ \\
\hline Multifocal & $5(16.7)$ \\
\hline Tumor size (invasive) after NAC (cm) & $1.9(2.3)$ \\
\hline
\end{tabular}




\begin{tabular}{|c|c|}
\hline Characteristics & Value \\
\hline Presence of in situ carcinoma after NAC & $14(46.7)$ \\
\hline Tumor size (in situ) after NAC (cm) & $3.2(3.3)$ \\
\hline Extensive intraductal component after NAC & $3(10.0)$ \\
\hline Lymphovascular invasion after NAC & $7(23.3)$ \\
\hline \multicolumn{2}{|c|}{ Data are presented as mean (standard deviation) or number (\%). } \\
\hline \multicolumn{2}{|c|}{$\begin{array}{l}\text { AC, adriamycin/cyclophosphamide; AT, adriamycin/docetaxel; DCIS, ductal carcinoma in situ, HER2, } \\
\text { human epidermal growth factor receptor 2; HR, hormone receptor; IDC, invasive ductal carcinoma; ILC } \\
\text { invasive lobular carcinoma; LCIS, lobular carcinoma in situ; NAC, neoadjuvant chemotherapy; pCR, } \\
\text { pathologic complete response; T, docetaxel; TCHP, docetaxel/carboplatin/trastuzumab/pertuzumab; } \\
\text { TH; docetaxel/trastuzumab. }\end{array}$} \\
\hline
\end{tabular}

\section{Tumor size assessment}

Mean tumor size after NAC on hitopathology, CEDM and CEMRI are as follows; $1.89 \mathrm{~cm}$ (range: 0$12.0 \mathrm{~cm}$ ), $1.22 \mathrm{~cm}$ (range: $0-7.0 \mathrm{~cm}$ ) and $1.13 \mathrm{~cm}$ (range: $0-5.1 \mathrm{~cm}$ ). CEDM estimated tumor size to within $1 \mathrm{~cm}$ in 19 patients (63.3\%) and underestimated tumor size by more than $1 \mathrm{~cm}$ in 7 patients (23.4\%). CEMRI estimated final tumor size to within $1 \mathrm{~cm}$ in 18 patients $(60.0 \%)$ and underestimated tumor size by more than $1 \mathrm{~cm}$ in 9 patients (30.0\%) (Table 2). 
Table 2

Accuracy of CEDM and CEMRI $(n=30)$

Value

Tumor size after NAC (cm)

Pathologic

$1.89(0-12.0)$

CEDM

$1.22(0-7.0)$

CEMRI

$1.13(0-5.1)$

CEDM vs. path

CEDM overestimates path $(>1 \mathrm{~cm})$

CEDM underestimates path $(>1 \mathrm{~cm})$

7 (23.4)

CEDM within $1 \mathrm{~cm}$

19 (63.3)

CEMRI vs. path

CEMRI overestimates path $(>1 \mathrm{~cm})$

$3(10.0)$

CEMRI underestimates path $(>1 \mathrm{~cm})$

$9(30.0)$

CEMRI within $1 \mathrm{~cm}$

$18(60.0)$

CEDM vs. CEMRI

CEDM greater than CEMRI $(>1 \mathrm{~cm})$

$7(23.3)$

CEDM less than CEMRI $(>1 \mathrm{~cm})$

$3(10.0)$

CEDM within $1 \mathrm{~cm}$ of MRI

$20(66.7)$

Data are presented as mean (range) or number (\%).

CEDM, contrast-enhanced digital mammography; CEMRI, contrast-enhanced magnetic resonance imaging; NAC, neoadjuvant chemotherapy.

\section{Diagnostic performance of CEDM and CEMRI}

Concordance coefficient between tumor sizes from CEDM, CEMRI and histopathologic result was 0.202 (95\% Cl: -0.109-0.512) for CEDM and 0.243 (95\% Cl: -0.035-0.520) for CEMRI (Table 3). Pearson correlation was 0.231 for CEDM $(p=0.220)$ and 0.303 for CEMRI $(p=0.104)$. Mean differences between CEDM and CEMRI and residual pathological tumor size were $0.668 \mathrm{~cm}(\mathrm{SD}=2.699)$ and $0.765 \mathrm{~cm}(\mathrm{SD}=$ 2.511), respectively. The differences between CEDM, CEMRI, and residual pathological tumor size are shown in Fig. 1 using Bland-Altman plots. Limits of agreement between pathologically evaluated tumor size and CEDM and CEMRI were $-4.621-5.957 \mathrm{~cm}$ and $-4.156-5.686 \mathrm{~cm}$, respectively. 
Table 3

Concordance of CEM and CEMRI with its agreement of tumor size after NAC

\section{Concordance $(95 \% \mathrm{Cl}) \quad$ Correlation ( $p$ value) Mean difference (SD) LOA}

$\begin{array}{lllll}\text { CEDM } & 0.202(-0.109-0.512) & 0.231(0.220) & 0.668(2.699) & -4.621-5.957 \\ \text { CEMRI } & 0.243(-0.035-0.520) & 0.303(0.104) & 0.765(2.511) & -4.156-5.686\end{array}$

CEDM, contrast-enhanced digital mammography; CEMRI, contrast-enhanced magnetic resonance imaging; $\mathrm{Cl}$, confidence interval; LOA, limit of agreement; SD, standard deviation.

Sensitivity for identifying residual lesion was higher for CEMRI $(66.7 \%, 95 \% \mathrm{CI} 44.7-84.4)$ than CEDM (62.5\%, 95\% Cl 40.6-81.2) (Table 4). CEDM's specificity for identifying residual lesion was $83.3 \%$ (95\% Cl 65.9-99.6), which was better than that of CEMRI (66.7\%, 95\% Cl 22.3-95.7). The PPV for detecting remnant lesion was $93.8 \%$ (95\% $\mathrm{Cl} 69.8-99.8)$ for CEDM and 88.9\% (95\% Cl 65.3-98.6) for CEMRI, and the NPV was $35.7 \%$ (95\% Cl 12.8-64.9) for CEDM and 33.3\% for CEMRI (95\% Cl 9.9-65.1) (Table 4).

Table 4

Sensitivity, specificity, PPV, and NPV of CEDM and CEMRI for measuring tumor size after NAC

\begin{tabular}{|lllll|}
\hline & $\begin{array}{l}\text { Sensitivity } \\
(\mathbf{9 5 \%} \mathrm{Cl})\end{array}$ & $\begin{array}{l}\text { Specificity } \\
\mathbf{( 9 5 \% ~ C l )}\end{array}$ & $\begin{array}{l}\text { PPV } \\
\mathbf{( 9 5 \% ~ C l )}\end{array}$ & $\begin{array}{l}\text { NPV } \\
\mathbf{( 9 5 \% ~ C l )}\end{array}$ \\
\hline CEDM & $62.5(40.6-81.2)$ & $83.3(65.9-99.6)$ & $93.8(69.8-99.8)$ & $35.7(12.8-64.9)$ \\
\hline CEMRI & $66.7(44.7-84.4)$ & $66.7(22.3-95.7)$ & $88.9(65.3-98.6)$ & $33.3(9.9-65.1)$ \\
\hline $\begin{array}{l}\text { CEDM, contrast-enhanced digital mammography; CEMRI, contrast-enhanced magnetic resonance } \\
\text { imaging; PPV, positive predictive value; NPV, negative predictive value; Cl, confidence interval. }\end{array}$ \\
\hline
\end{tabular}

\section{Discussion}

NAC is used to test response or resistance of chemotherapeutic agent and reduce micrometastatic diseases $[16,17]$. Evaluating tumor response after NAC is important for determining future treatment plans and predicting long-term outcomes. Currently, CEMRI is the imaging modality for response monitoring [18]. CEMRI yields superior outcomes compared to mammography and US for identifying residual disease following NAC $[19,20]$. However, CEMRI has several limitations, such as long exam time, high cost, low accessibility, and longer interpretation time. In comparison, CEDM is known for shorter procedure time, cheaper price, easy interpretability, shorter interpretation time, and no claustrophobia risk $[10,21,22]$. Also, female patients do need to schedule their imagining appointment according to menstrual cycle. Some clinicians have suggested to conduct CEDM and CEMRI according to menstrual phase; however, some argue no clear pattern in background parenchymal enhancement variation across the menstrual cycle for either CEDM or CEMRI [23]. CEDM also revealed equivalent result for detecting primary and multifocal cancers and estimating extent of cancers compared to CEMRI [5]. 
In present study, CEDM was evaluated for detecting and measuring remnant tumors after NAC. Although CEDM underestimated remnant tumor size in some patients, the difference between CEDM and pathologic results was less than $1 \mathrm{~cm}$ in $63.3 \%$ of cases. Barra et al. [24] reported similar results, with a difference between CEDM and pathologic size within $1 \mathrm{~cm}$ in almost $70 \%$ of cases. Limits of agreement with the residual tumor were lower for CEMRI than for CEDM, which was consistent with that found by Patel and colleagues [6].

Regarding diagnostic performance, CEDM demonstrated better specificity, PPV, and NPV compared to CEMRI, though CEMRI had better sensitivity than CEDM. Similarly, other studies reported comparable specificity and PPV for CEDM $[6,25]$. All women with radiologic CR on CEMRI had negative CEDM results. Our results also showed good correlation and concordance of CEDM with histopathologic result after NAC (Figs. 2 and 3, Table 3).

CEDM and CEMRI both underestimated the extent of remnant cancers (mean tumor sizes from CEDM, CEMRI, and pathology were $1.22 \mathrm{~cm}, 1.13 \mathrm{~cm}$, and $1.89 \mathrm{~cm}$, respectively). lotti et al. [26] also reported underestimation of tumor size by CEDM and CEMRI after NAC. Wu et al. [27] explained this discrepancy as an antiangiogenic effect of chemotherapeutic agents used in CEDM and CEMRI, such as taxanes and anthracyclines. This anti-vascular effect, which lacks a surrounding inflammatory response, lowers enhancement of tumor tissue and, thus, can result in tumor underestimation [20].

Limitations of the present study are associated several factors. First, we enrolled a small number of patients. This was possibly because patients lacked an understanding of CEDM. Since CEDM was recently introduced in South Korea, patients were not familiar with it, and most refused to undergo additional mammography because of the pain induced by breast compression during the procedure. Second, the ability to detect residual ductal carcinoma in situ (DCIS) or suspicious microcalcifications remains unknown for both CEDM and CEMRI. CEDM and CEMRI identify occult breast cancer using mechanism of tumor angiogenesis [28]; however, the underlying mechanism of tumor angiogenesis depends on subtype of breast cancer. Neo-angiogenesis is more prominent in IDC and is not always exist in DCIS and lobular carcinomas [29]. In our study, none of pCR patients showed residual DCIS component on residual tumor. The feasibility of CEDM for DCIS should be further evaluated. Third, we could not differentiate the diagnostic accuracy of CEDM according to presence or absence of microcalcifications. Finally, we could not assess the accuracy of CEDM according to molecular subtype, and further investigations are needed.

Our study also has several strengths. First, this is the first CEDM study conducted among breast cancer patients who received NAC in South Korea.. Second, we found that CEDM is secure and practical. There was no technical failures during procedure and one patient had side effects during CEDM examinations. The patient vomiting one minutes following injection of contrast media. The symptoms disappeared soon. Finally, because this was a prospective study, there was no inherent bias.

\section{Conclusions}


In conclusion, CEDM showed diagnostic results comparable to CEMRI in demonstrating residual cancers and estimating residual tumor extent after NAC. CEDM can be as effective as CEMRI for evaluating NAC patients.

\section{Abbreviations}

AC: Adriamycin/Cyclophosphamide; ACR: American College of Radiology; AT: Adriamycin/Docetaxel; BIRADS: Breast Imaging Reporting and Data System; CC: Craniocaudal; CEDM: Contrast-Enhanced Digital Mammography; CEMRI: Contrast-Enhanced Magnetic Resonance Imaging; Cls: Confidence intervals; CR: Complete response; DCIS: Ductal Carcinoma In Situ; ER ; Estrogen Receptor: FN: False Negative; FP: FalsePositive; HER-2 : Human Epidermal Growth factor Receptor-2; HR : Hormone Receptor; IDC: Invasive Ductal Carcinoma; ILC: invasive lobular carcinoma; LCIS: Lobular Carcinoma in situ; LOA: Limit Of Agreement; MIP: Maximum Intensity Projection; MLO: Medioateral oblique; NAC : Neoadjuvant chemotherapy; NOS : Not otherwise specified; NPV: Negative predictive value; $\mathrm{pCR}$ : pathologic complete response; PD : Progressive disease; PPV : Positive predictive value; PR : Partial response; PR :

Progesterone receptor; rCR : radiologic CR; RECIST : response evaluation criteria in solid tumors; SD : standard deviation; SD : stable disease; T: docetaxel; TCHP : docetaxel/carboplatin/trastuzumab/pertuzumab; TH: docetaxel/trastuzumab; TN : true negative; US : ultrasonography

\section{Declarations}

\section{Ethics approval and consent to participate}

This single-institution prospective study was approved by the Institutional Review Board Committee of Kangbuk Samsung Hospital, Seoul, Republic of Korea (no. 2017-09-027). Informed consent was obtained from all patients by written document.

\section{Consent for publication}

Not applicable

\section{Availability of data and materials}

The datasets used and/or analyzed during the current study are available from the corresponding author on reasonable request.

\section{Competing interest}

The authors declare that they have no competing interests. 


\section{Funding}

This work was supported by the Medical Research Funds from Kangbuk Samsung Hospital.

The funder had no role in the design of the study and collection, analysis, and interpretation of data and in writing the manuscript.

\section{Authors' contributions}

All authors have read and approved the manuscript.

EYK made a substantial contribution to the concept and design of the work.

JSY approved the version to be published.

YLP drafted the article and revised it critically for important intellectual content.

CHP have participated sufficiently in the work to take public responsibility for appropriate portions of the content.

MRK made a substantial contribution to the analysis of data.

JHM made a substantial contribution to the interpretation of data.

IYY drafted the article or revised it critically for important intellectual content.

YJC approved the version to be published.

SHK made a substantial contribution to the concept or design of the work.

\section{Acknowledgments}

The authors express special gratitude to mammography specialist technician Yeon-Sun Kim.

\section{Authors' information}

As corresponding author, Shin Ho Kook declare that all listed authors have fulfilled all 4 requirements for authorship according to international rules.

Name : Shin Ho Kook

Place : Department of Radiology, Kangbuk Samsung Hospital, Sungkyunkwan University School of Medicine 
Date : July, $23^{\text {rd }}, 2020$

\section{References}

1. Lewin JM, Isaacs PK, Vance V, Larke FJ: Dual-energy contrastenhanced digital subtraction mammography: feasibility. Radiology 2003, 229(1):261-268.

2. Fallenberg EM, Dromain C, Diekmann F, Engelken F, Krohn M, Singh JM, Ingold-Heppner B, Winzer KJ, Bick U, Renz DM: Contrast-enhanced spectral mammography versus MRI: Initial results in the detection of breast cancer and assessment of tumour size. European radiology 2014, 24(1):256-264.

3. Dromain C, Thibault F, Muller S, Rimareix F, Delaloge S, Tardivon A, Balleyguier C: Dual-energy contrastenhanced digital mammography: initial clinical results. European radiology 2011, 21(3):565574.

4. Bilimoria KY, Cambic A, Hansen NM, Bethke KP: Evaluating the impact of preoperative breast magnetic resonance imaging on the surgical management of newly diagnosed breast cancers. Arch Surg 2007, 142(5):441-447.

5. Lee-Felker SA, Tekchandani L, Thomas M, Gupta E, Andrews-Tang D, Roth A, Sayre J, Rahbar G: Newly Diagnosed Breast Cancer: Comparison of Contrastenhanced Spectral Mammography and Breast MR Imaging in the Evaluation of Extent of Disease. Radiology 2017, 285(2):389-400.

6. Patel BK, Hilal T, Covington M, Zhang N, Kosiorek HE, Lobbes M, Northfelt DW, Pockaj BA: ContrastEnhanced Spectral Mammography is Comparable to MRI in the Assessment of Residual Breast Cancer Following Neoadjuvant Systemic Therapy. Ann Surg Oncol 2018, 25(5):1350-1356.

7. Barra FR, Sobrinho AB, Barra RR, Magalhaes MT, Aguiar LR, de Albuquerque GFL, Costa RP, Farage L, Pratesi R: Contrast-Enhanced Mammography (CEM) for Detecting Residual Disease after Neoadjuvant Chemotherapy: A Comparison with Breast Magnetic Resonance Imaging (MRI). BioMed research international 2018, 2018:8531916.

8. The Korean Society of Radiology, The Korean Academy of Asthma, Allergy and Clinical Immunology: Korean clinical practice guideline for adverse reactions to injectable iodinated contrast agent and gadolinium-based contrast agent injection for MRI. In., 2nd edn. Seoul: The Korean Society of Radiology; 2016.

9. Ren B, Ruth C, Zhang Y, Smith A, Kennedy D, O'Keefe B, Shaw I, Williams C, Ye Z, Ingal E et al: Dual energy iodine contrast imaging with mammography and tomosynthesis. In: SPIE Medical Imaging: 2013 Feb 9-14 2013; Lake Buena Vista, FL: SPIE 2013: 86680U.

10. Kim EY, Youn I, Lee KH, Yun JS, Park YL, Park CH, Moon J, Choi SH, Choi YJ, Ham SY et al: Diagnostic Value of Contrast-Enhanced Digital Mammography versus Contrast-Enhanced Magnetic Resonance Imaging for the Preoperative Evaluation of Breast Cancer. J Breast Cancer 2018, 21(4):453-462.

11. American College of Radiology, BI-RADS Committee: ACR BI-RADS Atlas: Breast Imaging Reporting and Data System, 5th edn. Reston, VA: American College of Radiology; 2013. 
12. Eisenhauer EA, Therasse P, Bogaerts J, Schwartz LH, Sargent D, Ford R, Dancey J, Arbuck S, Gwyther $\mathrm{S}$, Mooney $\mathrm{M}$ et al: New response evaluation criteria in solid tumours: revised RECIST guideline (version 1.1). Eur J Cancer 2009, 45(2):228-247.

13. Kim EY, Do SI, Yun JS, Park YL, Park CH, Moon JH, Youn I, Choi YJ, Ham SY, Kook SH: Preoperative evaluation of mammographic microcalcifications after neoadjuvant chemotherapy for breast cancer. Clin Radiol 2020, 75(8):641.e619-641.e627.

14. Harvey JM, Clark GM, Osborne CK, Allred DC: Estrogen receptor status by immunohistochemistry is superior to the ligand-binding assay for predicting response to adjuvant endocrine therapy in breast cancer. Journal of clinical oncology : official journal of the American Society of Clinical Oncology 1999, 17(5):1474-1481.

15. von Minckwitz G, Untch M, Blohmer JU, Costa SD, Eidtmann H, Fasching PA, Gerber B, Eiermann W, Hilfrich J, Huober $\mathrm{J}$ et al: Definition and impact of pathologic complete response on prognosis after neoadjuvant chemotherapy in various intrinsic breast cancer subtypes. Journal of clinical oncology : official journal of the American Society of Clinical Oncology 2012, 30(15):1796-1804.

16. Ogston KN, Miller ID, Payne S, Hutcheon AW, Sarkar TK, Smith I, Schofield A, Heys SD: A new histological grading system to assess response of breast cancers to primary chemotherapy: prognostic significance and survival. Breast 2003, 12(5):320-327.

17. Mieog JSD, van der Hage JA, van de Velde CJH: Preoperative chemotherapy for women with operable breast cancer. Cochrane Database Syst Rev 2007, 2007(2):CD005002.

18. Shin HJ, Kim HH, Ahn JH, Kim SB, Jung KH, Gong G, Son BH, Ahn SH: Comparison of mammography, sonography, MRI and clinical examination in patients with locally advanced or inflammatory breast cancer who underwent neoadjuvant chemotherapy. Br J Radio/2011, 84(1003):612-620.

19. Martincich L, Montemurro F, De Rosa G, Marra V, Ponzone R, Cirillo S, Gatti M, Biglia N, Sarotto I, Sismondi $\mathrm{P}$ et al: Monitoring response to primary chemotherapy in breast cancer using dynamic contrast-enhanced magnetic resonance imaging. Breast Cancer Res Treat 2004, 83(1):67-76.

20. Lobbes MBI, Prevos R, Smidt M, Tjan-Heijnen VCG, van Goethem M, Schipper R, Beets-Tan RG, Wildberger JE: The role of magnetic resonance imaging in assessing residual disease and pathologic complete response in breast cancer patients receiving neoadjuvant chemotherapy: a systematic review. Insights Imaging 2013, 4(2):163-175.

21. Hobbs MM, Taylor DB, Buzynski S, Peake RE: Contrast-enhanced spectral mammography (CESM) and contrast enhanced MRI (CEMRI): Patient preferences and tolerance. Journal of medical imaging and radiation oncology 2015, 59(3):300-305.

22. Youn I, Choi S, Choi YJ, Moon JH, Park HJ, Ham S-Y, Park CH, Kim EY, Kook SH: Contrast enhanced digital mammography versus magnetic resonance imaging for accurate measurement of the size of breast cancer. Br J Radiol 2019, 92(1098):20180929.

23. Sogani J, Morris EA, Kaplan JB, D'Alessio D, Goldman D, Moskowitz CS, Jochelson MS: Comparison of Background Parenchymal Enhancement at Contrast-enhanced Spectral Mammography and 
Breast MR Imaging. Radiology 2017, 282(1):63-73.

24. Barra FR, Sobrinho AB, Barra RR, Magalhães MT, Aguiar LR, de Albuquerque GFL, Costa RP, Farage $L$, Pratesi R: Contrast-Enhanced Mammography (CEM) for Detecting Residual Disease after Neoadjuvant Chemotherapy: A Comparison with Breast Magnetic Resonance Imaging (MRI). Biomed Res Int 2018, 2018:8531916.

25. EISaid NAE, Mahmoud HGM, Salama A, Nabil M, EIDesouky ED: Role of contrast enhanced spectral mammography in predicting pathological response of locally advanced breast cancer post neoadjuvant chemotherapy. Egypt J Radiol Nucl Med 2017, 48(2):519-527.

26. Iotti V, Ravaioli S, Vacondio R, Coriani C, Caffarri S, Sghedoni R, Nitrosi A, Ragazzi M, Gasparini E, Masini $C$ et al: Contrastenhanced spectral mammography in neoadjuvant chemotherapy monitoring: a comparison with breast magnetic resonance imaging. Breast Cancer Res 2017, 19(1):106.

27. Wu L-A, Chang R-F, Huang C-S, Lu Y-S, Chen H-H, Chen J-Y, Chang Y-C: Evaluation of the treatment response to neoadjuvant chemotherapy in locally advanced breast cancer using combined magnetic resonance vascular maps and apparent diffusion coefficient. J Magn Reson Imaging 2015, 42(5):1407-1420.

28. Dromain C, Thibault F, Diekmann F, Fallenberg EM, Jong RA, Koomen M, Hendrick RE, Tardivon A, Toledano A: Dual-energy contrast-enhanced digital mammography: initial clinical results of a multireader, multicase study. Breast Cancer Res 2012, 14(3):R94.

29. Kuhl CK: MRI of breast tumors. Eur Radiol 2000, 10(1):46-58.

\section{Figures}

a

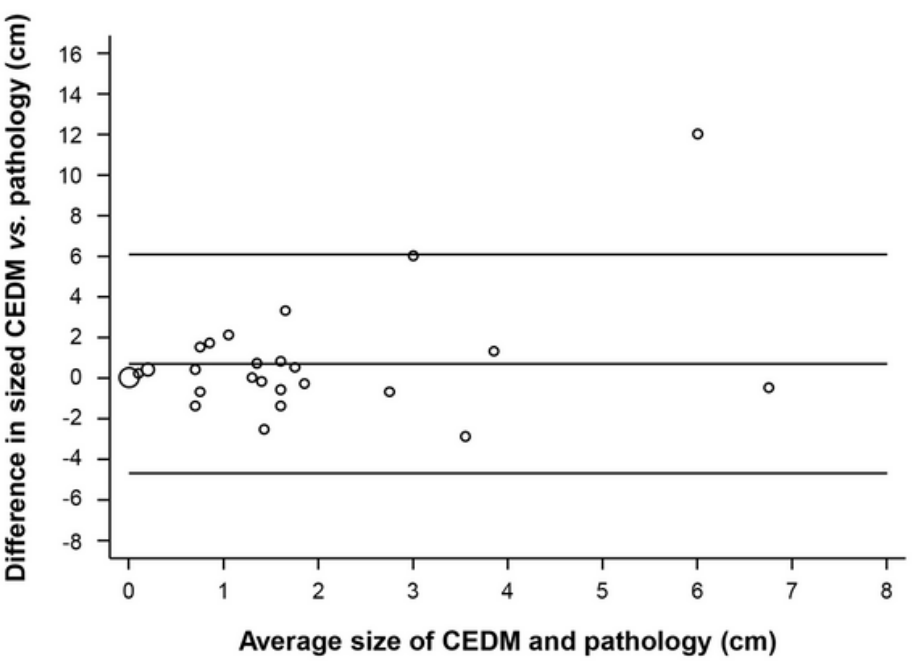

b

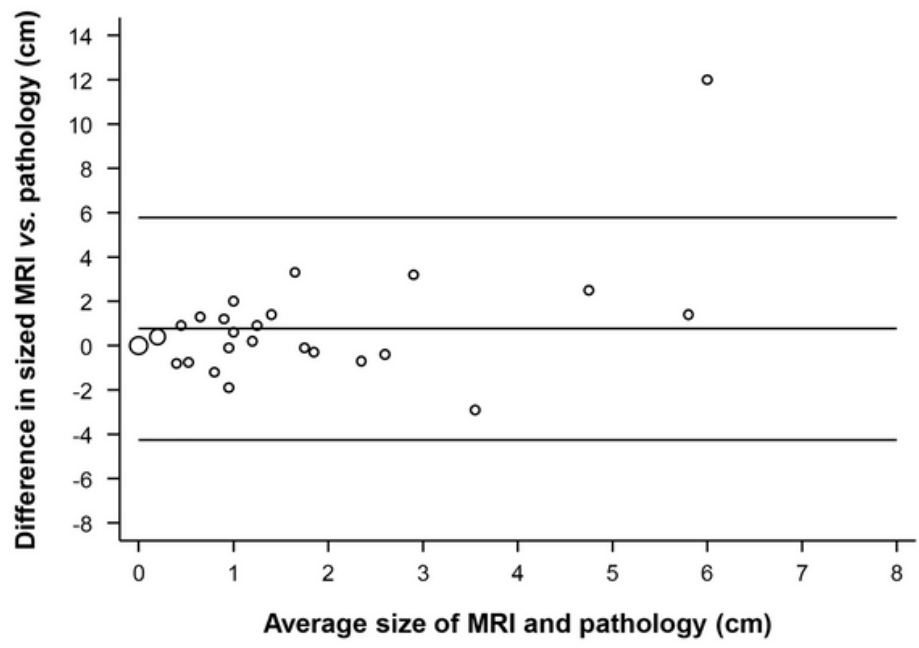

Figure 1

Correlation between histopathologic tumor size and CEDM (a) and CEMRI (b) described by Bland-Altman plots. We set $-1 \mathrm{~cm}$ to $1 \mathrm{~cm}$ as the lower and upper limit for the equivalence test, i.e., if the difference 
between CEDM/CEMRI measurements. If the result was within this boundary, we confirmed the result to be equivalent to the histopathological result.
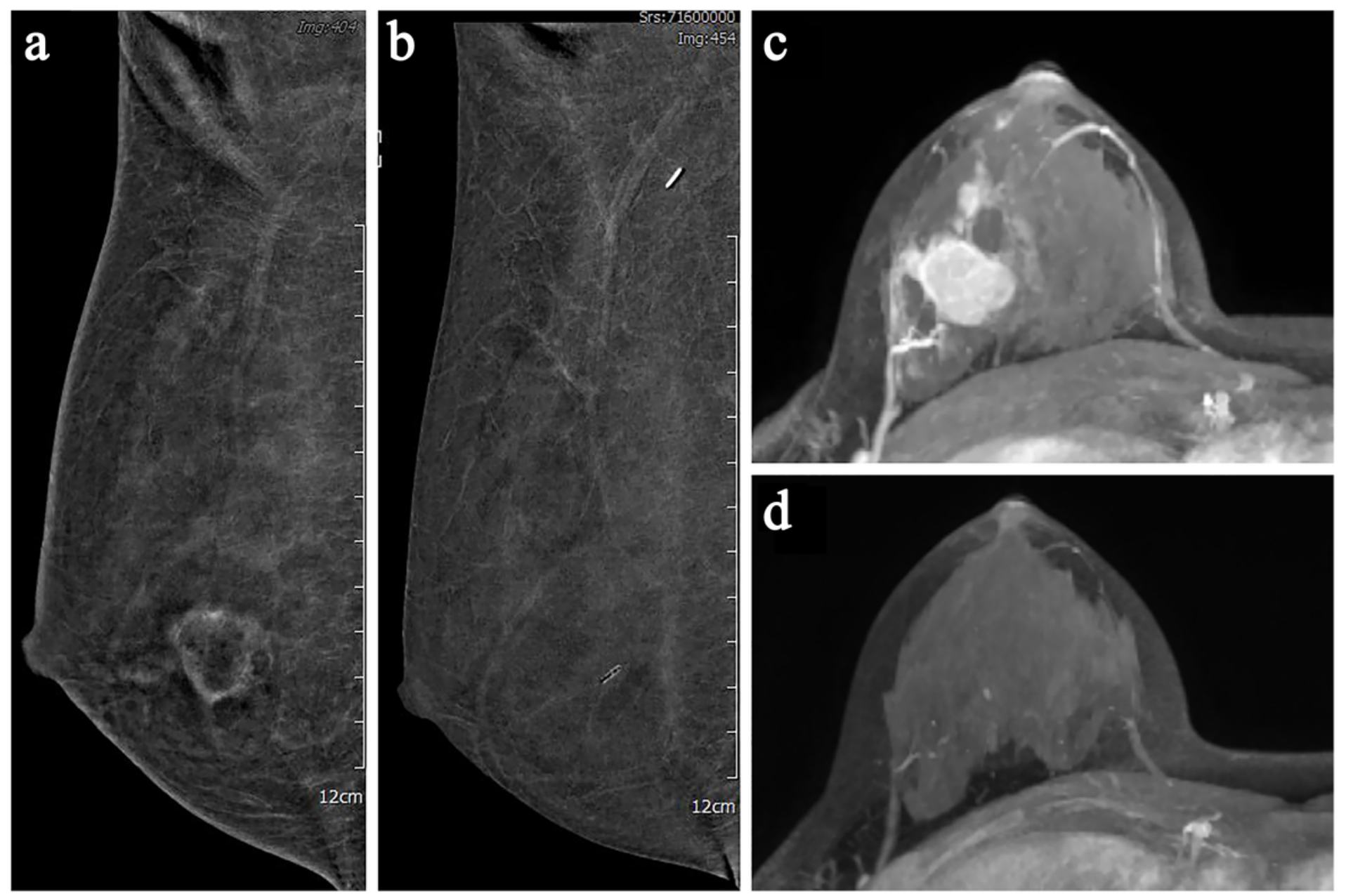

\section{Figure 2}

Pathologic complete response in a 31-year-old woman with IDC in lower center quadrant of the right breast, correctly assessed using CEDM and CEMRI (maximum intensity projection [MIP] image). Before NAC, CEDM revealed a rim enhanced mass accompanying scattered small enhancing foci (a; mediolateral oblique [MLO] image). CEMRI revealed an enhancing mass (c; MIP view). After NAC, there was no visible enhancement on either CEDM (b; MLO image) or CEMRI (d; MIP view). Pathologic complete response was achieved. 

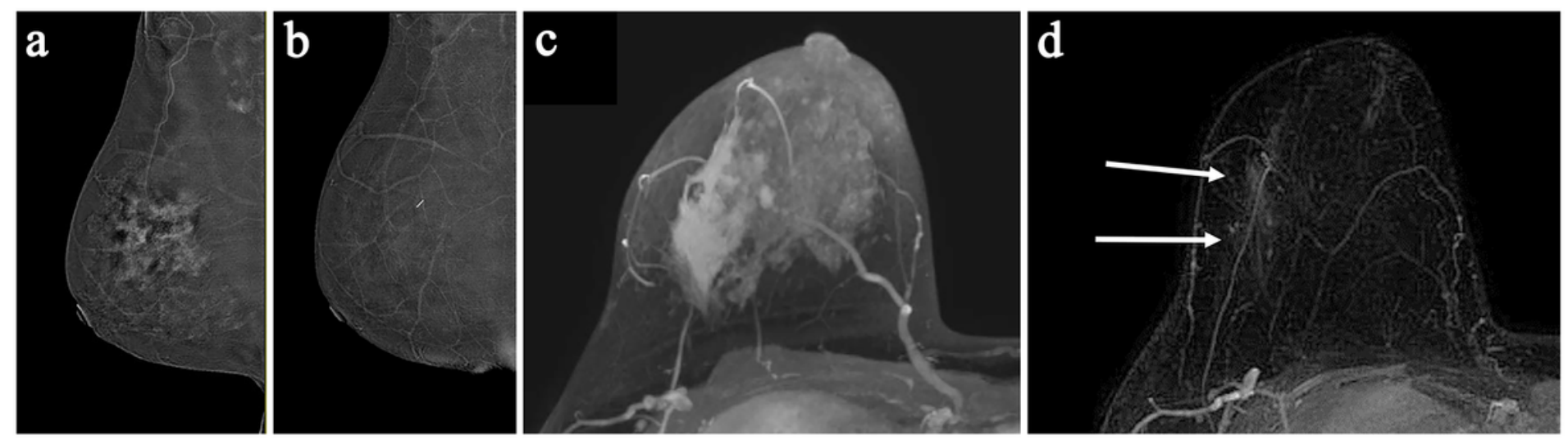

\section{Figure 3}

Pathologic complete response in a 38-year-old woman with IDC in upper outer quadrant of the right breast, correctly assessed using CEDM but not CEMRI. Before NAC, CEDM revealed an irregular heterogeneous enhancing area (a; MLO image). CEMRI revealed an enhancing lesion with a multicentric, conglomerate irregular pattern with accompanying tiny daughter nodules (c; MIP view). After NAC, there was no visible enhancement on CEDM (b; MLO image). There was a non-mass enhancement area (arrows) at the outer peripheral aspect of a previously noted main mass on CEMRI (d; MIP view). Pathologic complete response was achieved. 